\title{
PRP: review of the current evidence for musculoskeletal conditions
}

\author{
Gerard A. Malanga $\cdot$ Michael Goldin
}

Published online: 25 January 2014

(c) Springer Science + Business Media New York 2014

\begin{abstract}
Injection of platelet-rich plasma (PRP) is an evolving treatment option for various musculoskeletal injuries. There is basic scientific evidence that suggests that the various growth factors present in PRP can help to augment the body's natural healing. There are also clinical studies suggesting efficacy for several conditions, particularly tendinopathy and osteoarthritis. This article reviews the definition and first uses of PRP, the basic scientific rationale for its use, and the basic science and evidence for its use in the treatment of tendon, joint, ligament, and muscle injuries. There are varying levels of evidence for and against the use of PRP for these types of injuries, and this article reviews studies that support as well as studies that refute the use of this new treatment. There are several studies that have assessed the basic science supportive of PRP treatments, as well as the clinical efficacy of this treatment in vivo. While the current evidence is mixed, several recent studies have demonstrated therapeutic benefit in the treatment of various tendinopathies and degenerative joint diseases of the knee. There are several factors that need to be addressed to elucidate whether PRP is truly effective. These include fully defining the PRP mixture (e.g. concentration, growth factor levels, presence of white cells and red cells, etc.), determining the optimal preparation and delivery of the PRP graft, calculating the
\end{abstract}

G. A. Malanga $\cdot$ M. Goldin

New Jersey Sports Medicine, LLC, New Jersey Regenerative Institute, New Brunswick, NJ, USA

G. A. Malanga $(\bowtie) \cdot$ M. Goldin

Department of Physical Medicine and Rehabilitation, Rutgers

University-New Jersey Medical School, New Brunswick, NJ, USA

e-mail: gerardmalanga@gmail.com appropriate number of injections for each specific pathologic process, and defining optimal post-procedure rehabilitation.

Keywords PRP · Tendinopathy · Treatment · Arthritis · Growth factors

\section{Introduction}

Platelet-rich plasma (PRP) has evolved as a treatment option for a variety of orthopedic conditions over the past decade. PRP treatments have historically been used in cardiac and dental procedures in humans. Since these initial uses, it has been used in a variety of musculoskeletal conditions in humans over the past 10 years. Initial studies were directed at treatment of chronic, refractory tendinopathy, in particular chronic tennis elbow pain. Over the past decade, its use has been expanded to treating a variety of other musculoskeletal conditions including ligament injuries, muscle tears, and osteoarthritis.

In this article, we will review the current evidence regarding PRP for musculoskeletal injuries. We will first review the basic science of PRP and the rationale for its use in tendinopathy and other musculoskeletal conditions. This review will primarily focus on the evidence specifically for non-invasive management of pathologic processes involving tendons, joints, ligaments, and fibro-cartilaginous structures.

\section{Definition}

PRP has been defined as a volume of autologous plasma that has a platelet concentration above baseline [1]. The 
process of concentrating the platelets necessitates two sets of centrifugation, one after the other. The first spin (a hard spin) separates the red blood cells from the plasma, the latter of which contains platelets, white blood cells, and clotting factors [1]. The second spin (a soft spin) finely separates the platelets and white blood cells, together with a few red blood cells [1]. In 2003, Weibrich et al. [2] felt that the optimum platelet concentration in PRP to have a positive effect on bone regeneration was around 1 million platelets/microliter $(\mu \mathrm{l})$, and above that, there was an inhibitory effect on healing. However, several recent articles have produced different conclusions about ideal platelet count and challenged these initial theories. Giusti et al. found the optimal platelet concentration for angiogenesis was 1.5 million/ $\mu \mathrm{l}(5-7 \times$ baseline). Lower levels produced less angiogenesis, and inhibition was not noted until levels reached 2-3 million/ $\mu$ l $(10 \times$ baseline) [3]. Finally, Kevy documented that the ideal platelet concentration is 1.5 million/ $\mu \mathrm{l}(5-7 \times$ baseline $)$ and could be as high as 3 million/ $\mu \mathrm{l}$ ( $10 \times$ base-line). Kevy and Jacobson [4] also noted that current commercially available PRP devices could not attain levels higher than $10 \times$ baseline levels.

\section{First uses}

In 1987, Ferrari et al. [5] used autologous PRP and red blood cell concentrates as an autologous transfusion for support of cardiac surgery patients in 15 operations. No homologous blood products were required. In 1990, DelRossi et al. [6] showed that in patients undergoing heart operations on cardiopulmonary bypass, PRP-infused patients required $65 \%$ less banked blood products compared to patients not receiving this infusion. In 1998, Marx et al. [7] assessed PRP and its effect on increasing the rate of bone graft maturity of dental implants compared to a control group. This study demonstrated that an average concentration of $338 \%$ of baseline platelet count resulted in greater trabecular bone density relative to bone grafts that were not augmented with PRP [7].

\section{Rationale for use of PRP}

Platelets have several components that may help to augment healing. Dense granules within the platelets have compounds that influence cell migration, cell proliferation, and vascular tone [8]. They contain several types of granules, each with different components and various roles in platelet activities. These granules include dense granules, alpha granules, lysosomal granules, etc. Alpha granules have several proteins with various functions including platelet-derived growth factors (PDGF) such as $P D G F \alpha \alpha$, $P D G F \beta \beta$, and $P D G F \alpha \beta ; T G F \beta 1$ and TGF 2 ; vascular endothelial growth factor (VEGF), transforming growth factor beta (TGFB), insulin-like growth factor (IGF), vascular endothelial growth factor (VEGF), basic fibroblast growth factor (BFGF), and epithelial growth factor (EGF). The functional groupings of these proteins include adhesive proteins, clotting factors, fibrinolytic factors, proteases, growth factors, cytokines, basic proteins, antimicrobial proteins, and membrane glycoproteins [8].

PDGF and epidermal growth factors are present in tendons during the tendon healing process [9]. TGF $\beta$ has been shown to increase type I and type III collagen production in tendon sheath cells, epitenon cells, and endotenon cells [10]. Marui et al. [11] showed that TGF $\beta-1$ increased collagen and non-collagen protein synthesis in the medial collateral ligament and anterior cruciate ligament fibroblasts in a dosedependent manner. Koch et al. [12] studied TGF $\beta-1$ in mice, finding that the release of this growth factor from platelets and macrophages may actually increase inflammation and slow wound closure. In the early stages of inflammation, matrix metalloproteinases activate TGF $\beta$ - 1 from macrophages, which acts as a chemoattractant for neutrophils [11] and, later on in the process, promotes tissue repair [13].

In contrast, in a study using a mouse model, TGF $\beta-3$ was found to progressively decrease during the onset of osteoarthritis [14]. However, TGF $\beta-3$ was found to be strongly expressed in chondrocyte clusters just prior to osteophyte formation [12]. In a human model, Verdier et al. [15] demonstrated that TGF $\beta-1$ had decreased expression in degraded cartilage. However, in osteophytes, there was a marked increase in expression of TGF $\beta-1$ and TGF $\beta-3$. This research suggests that these growth factors have different functions depending on the stage of degeneration of the joint. Marui et al. [11] also showed that BFGF administration was not associated with an increase in collagen or non-collagen protein synthesis.

VEGF has been shown to be increased by day 7 after acute tendon injury, and new vessel length and density has been shown to peak at 17 days after acute injury [16]. Daily injections of epidermal growth factor have been shown to increase the extent and organization of granulation tissue [17]. IGF has been shown to increase the synthesis of proteoglycan, collagen, and non-collagen proteins [18]. Phornphutkul et al. [19] showed that IGF-1 promotes chondrocyte proliferation and differentiation.

\section{PRP duration of effect}

Prior literature has suggested that once platelets are activated by clotting, $70 \%$ of the stored growth factors are released after $10 \mathrm{~min}$, and almost all stored growth factors 
are released within $1 \mathrm{~h}$ of injection [1]. The platelets can then synthesize and secrete growth factors for about 8 days, until the platelets die [1].

Various factors should be weighed when considering the use of PRP and when reviewing prior research of PRP. These include platelet concentration, leukocyte count, $\mathrm{pH}$ of the injected substance, use of activators, the total number of injections given, and the method of delivery and the type of post-procedure rehabilitation protocol [20]. In particular, the post-procedure rehabilitation has ranged from activities as tolerated to full immobilization. A suggested post-rehabilitation program was present in the paper based on the known healing patterns of most tissues with progressive loading of the tissues through the various phases following PRP procedures. This generally allows for full activities at the 6-8 week time-frame following injection [20].

The components of PRP have also been examined to assess if certain compositions have a better healing profile. Dragoo et al. [21] showed that leukocyte-rich PRP had a greater inflammatory response relative to leukocyte-poor PRP at post-injection day 5, but there was no difference at day 14. McCarrel et al. [22] demonstrated that leukocytereduced PRP had decreased levels of tumor necrosis factor alpha and interleukin 1 beta relative to standard PRP, highconcentration PRP, and concentrated-leukocyte PRP.

\section{Evidence regarding use of PRP for tendinopathy}

\section{Basic science}

De Mos et al. [23] showed that both platelet-rich clot releasate (PRCR) and platelet-poor clot releasate (PPCR) increased tenocyte cell number and collagen production in vitro. In this study, PRP was "activated" by placing calcium chloride into the PRP concentrate to induce the platelets to clot and to release their growth factors. Some limitations of this study are that the tendons were from young donors, and the fact that this experiment was performed in vitro and, therefore, may not be applicable in vivo. Additionally, this PRCR was not autologous, but rather the PRCR was from a different donor source than the tendons. Wang et al. [24] demonstrated that PRCR can promote human tenocyte proliferation and promote collagen synthesis. Bosch et al. [25] performed a study in which they surgically created lesions in the superficial digital flexor tendons of horses. They then used a commercially available PRP system, Biomet, to create $3 \mathrm{ml}$ of therapeutic injectate. Ultrasound guidance was used to place either the PRP or the control in the lesion. They demonstrated that there was statistically significant improvement in collagen, glycosaminoglycan, and DNA content in the PRP-treated tendons relative to the controls. In addition, the repair tissue showed higher strength failure and better collagen organization relative to the control tendons. One of the limitations of this study was that the lesion was created artificially by acutely inducing mechanical damage. This differs from the more clinically relevant incidence in humans in which repetitive overuse injury is primarily the etiology for tendon damage [25]. Zhang and Wang [26] showed that PRCR promotes the differentiation of tendon stem cells into active tenocytes. This experiment was performed in vitro, and used PRP that was activated with calcium chloride to form the PRCR. These tenocytes had increased collagen production relative to control cells treated only with autologous serum. Furthermore, this showed that PRCR did not induce cells to turn into "nontenocytes". This was significant, because it demonstrated that PRCR would not change tendon cells into non-tendon cells, potentially exacerbating a tendinopathy. Strengths of this study included that autologous serum was used on the tendon stem cells. Limitations of this study include that healthy tendon stem cells from young rabbits were used, and this may not be applicable to subjects with older tendons. Additionally, these experiments were performed in vitro; therefore, the tendons were not subjected to the mechanical loading that occurs in vivo [26]. Platelet concentrate was found to improve Achilles tendon repair callus strength for 3 weeks after surgical transection in an experimental rat model. This experiment used heterologous PRCR activated by thrombin. The PRCR was obtained from one group of rats and was injected into different rats with surgically induced lesions; thus, it was not autologous PRCR. Limitations of this study include the possibility that the measured force that caused failure of the un-operated tendons may have been underestimated due to the design of the mechanical testing apparatus [27]. Sadoghi et al. [28] studied whether rotator cuff fibroblasts isolated from human subjects would have dosedependent increased proliferation when exposed to PRP. They showed that a concentration of platelets five times that of plasma had an effect on increasing human rotator cuff fibroblast proliferation, specifically, increasing the DNA to glycosaminoglycan ratio (the latter being one of the markers for tendon degeneration). The onefold and five-fold concentrations of PRP had improved DNA to glycosaminoglycan ratios relative to the ten-fold concentration of PRP. The limitations of this study include the older age of the patients (61 years or older), which may have limited both the amount of growth factors present in the PRP and the regenerative response of (older) rotator cuff fibroblasts.

\section{Evidence regarding use of PRP for meniscus/cartilage}

Ishida et al. [29] demonstrated that PRP had regenerative effects on meniscal cells in vitro. The PRP was prepared by doing serial centrifugations at $4{ }^{\circ} \mathrm{C}$. Once the PRP was obtained, it was thawed and then stored at $-80{ }^{\circ} \mathrm{C}$ until ready for use. Furthermore, in vivo PRP combined with a hydrogel 
had beneficial healing effects on surgically induced meniscal lesions in a rabbit model. Limitations of this study include that the lesions were surgically induced, and may therefore not have similar results in primarily degenerative lesions [29]. Surgically induced osteochondral defects were treated in a rabbit model. A polylactic-glycolic acid scaffold was impregnated with PRP and a thrombin/calcium chloride solution to activate the PRP. This was then surgically implanted in the osteochondral defect. Activated PRPimpregnated polylactic glycolic acid improved osteochondral healing relative to a control group in a rabbit model. This study included the limitation that the osteochondral defect was surgically induced, and a traumatic or degenerative lesion may have a different response to this treatment [30]. Van Buul et al. [31] performed a laboratory study in which they examined whether PRP releasate (PRPr) could decrease the effect of interleukin-1 on osteoarthritic chondrocytes. The PRPr was formed by serial centrifugation of whole blood from healthy donors, and then activated with calcium chloride. PRPr decreased multiple inflammatory effects of IL-1 beta on human osteoarthritic chondrocytes in this in vitro study. Limitations of this study include that the PRPr was not autologous, and it is questionable whether these in vitro effects would occur in vivo. Akeda et al. [32] used PRPr activated with a thrombin/calcium chloride solution to examine effects on porcine chondrocytes in vitro. This PRPr administration to chondrocytes resulted in a statistically significant increase in collagen synthesis and chondrocyte DNA relative to chondrocytes treated with platelet-poor plasma releasate or fetal bovine serum. Additionally, the chondrocyte cells remained phenotypically stable in the presence of the PRP. Limitations of this study included the fact that the chondrocytes were pooled from multiple pigs, and therefore it was not purely autologous PRPr [32].

Evidence regarding use of PRP for muscle injury

In a mouse model, experimentally induced contusion to the gastrocnemius muscle was treated with a series of three injections of either autologous conditioned serum (ACS) or a series of three injections of saline [33]. Histological evaluation revealed that there was increased myofiber diameter regeneration and increased satellite cell activation in the ACS group compared to the control group [33].

\section{Evidence regarding use of PRP for tendinopathy}

\section{Lateral elbow/common extensor origin}

Mishra and Pavelko [34] examined a cohort of 20 patients with chronic lateral epicondylosis that had been refractory to a standardized physical therapy protocol and who had significant and persistent pain for an average of 15 months. These patients were all considering surgical treatment. Fifteen patients were given a single PRP injection, and five were given a single bupivacaine injection. The PRP injectate was produced by serial centrifugation of autologous whole blood. No activating agent was used, and the PRP was buffered to physiologic $\mathrm{pH}$ using $8.4 \%$ sodium bicarbonate solution. The tendon was anesthetized with $0.5 \mathrm{ml}$ of bupivacaine with epinephrine. The outcome measures that they used were a $100-\mathrm{mm}$ visual analog pain score ( 0 , no pain; 100 , worst pain possible) and a modified Mayo elbow score (best score, 100). At the 8-week followup, the PRP patients had $60 \%$ improvement in symptoms, and the bupivacaine group had $16 \%$ improvement in their symptoms. Three of the five control subjects withdrew or sought other treatments after 8 weeks. At 6 months' follow-up, the PRP cohort noted $81 \%$ improvement in the visual analog pain scores. At final follow-up (an average of 25.6 months), the PRP patients reported $93 \%$ pain reduction. The limitations of this study include the lack of a randomized control group, and the small number of patients. The tendon also had a small amount of bupivacaine injected, which can theoretically decrease the efficacy of PRP [35]. It is also important to note that 140 patients were initially evaluated, and only $15 \%$ were enrolled in the study. This may be viewed as one of the strengths of this study, as PRP was reserved as a treatment only for patients with severe tendinopathy that did not improve with either time or more conservative measures. Additional strengths include that all patients had also completed a standardized stretching and strengthening protocol prior to the injections, and after the injections all subjects were again given a standardized 4-week stretching and strengthening program [34].

Peerbooms et al. [36] compared PRP injections to corticosteroid injections in patients with greater than 6 months of pain from lateral epicondylitis. One hundred patients were randomized to receive either a PRP injection or a corticosteroid injection into the extensor tendon of the symptomatic elbow. The PRP preparation method involved serial centrifugations, buffering with sodium bicarbonate $8.4 \%$, and adding $0.5 \%$ bupivacaine with epinephrine to the injectate. Success was defined as $25 \%$ improvement in either VAS score or DASH (Disabilities of the arm, shoulder, and hand) score, without a re-intervention after 1 year. Using the VAS score, $49 \%$ of the corticosteroid group and $73 \%$ of the PRP group were successful. Using the DASH score, $51 \%$ of the corticosteroid group and $73 \%$ of the PRP group were successful. There were several strengths of this study. It was double-blind, randomized, and controlled. Patients were excluded if they had a steroid injection into the tendon within the past 6 months. The subjects were given a graded post-procedure 
rehabilitation including 2 weeks of stretching followed by 2 weeks of eccentric strengthening. The primary outcome measure, DASH, was a validated upper limb functional score. Limitations of this study include that the PRP was combined with a local anesthetic, which may inhibit some of the beneficial effects of the PRP [35]. Additionally, prior to the procedure, subjects had been treated with cast immobilization, a steroid injection, or physiotherapy. All of the subjects had not definitively undergone a course of physical therapy and were deemed to have symptoms recalcitrant to this [36]. This cohort of patients was also examined at a 2-year follow-up to assess efficacy of the intervention [37•]. At the 2-year follow-up, with success defined as $25 \%$ improvement in either VAS score or DASH score without a re-intervention after 2 years, $65 \%$ of the PRP group and $35 \%$ of the corticosteroid group had successful outcomes [37•].

In a prospective, double-blind, randomized trial, 150 patients were randomized to receive either two PRP injections or two autologous blood injections (ABIs) [38]. Successful outcome was defined as a 25-point improvement on the Patient-Rated Tennis Elbow Evaluation (PRTEE), which they reported was comparable to other studies. The authors reported that PRTEE is a well validated 0-100 composite scale measuring pain and physical function. At the 6-month follow-up, there was a $66 \%$ success rate in the PRP group and a $72 \%$ success rate in the ABI group, with no statistically significant difference between the two groups [34]. Strengths of this study include that all the patients had already failed a course of physical therapy including stretching and eccentric strengthening. They also had a single practitioner who had 15 years of experience and had performed 20,000 ultrasound guided injections perform all the injections. Limitations of this study include that the tendon was bathed in bupivacaine in both treatment groups, as this can potentially decrease the therapeutic effect [35]. Also, the performing physician was not blinded to the procedure. Additionally, this study introduces the experiment as comparing an ABI to a "moderate yield PRP" which they describe as "essentially plasma with erythrocytes and leukocytes removed". However, in the methods section, they describe obtaining the plasma by doing a single centrifugation and then obtaining $1.5 \mathrm{ml}$ from "the buffy coat layer". In the introduction, they had described the buffy coat as "leukocyte-rich, high yield PRP." Therefore, one of these descriptions is inaccurate. Another randomized controlled trial evaluated 28 patients who received either a single injection of PRP or a single injection of ABI. The PRP was obtained by performing a single centrifugation of the whole blood and then the platelet-rich portion was aspirated. In the analysis of the PRP, they described it as leukocyte-containing PRP due to histologic analysis showing leukocytes. Follow-up VAS scores were taken at 6 weeks, 3 months, and 6 months. Changes in VAS scores and Liverpool elbow scores were used as outcome measures. They report that the Liverpool elbow score evaluates range of motion, daily activities, and ulnar nerve function. There was improvement in both groups at all follow-up assessments. The only statistically significant difference between the two groups was a larger reduction in pain score in the PRP group compared to the ABI group at 6 weeks' follow-up. They did not report any clinically significant differences in the two groups [39]. Strengths of this study include that each subject was given a stretching and eccentric strengthening exercise program one week after the injection. Limitations of this study include that it was single-blind, and the patients were aware which treatment they were receiving. They also explain that the Liverpool elbow score evaluates elbow range of motion as well as the ulnar nerve, which is not usually affected in this condition. They explain that these two components may hide clinically significant differences.

Krogh et al. [40॰] performed a randomized controlled trial on 60 patients comparing the effects of a blinded injection of PRP, saline, or glucocorticoid. The primary outcome measure was a change in pain using the PRTEE questionnaire at 3 months post-procedure. For pain assessment, the PRTEE validated for lateral epicondylitis was applied. The PRP was processed by performing a single centrifugation cycle on whole blood, and then the PRP was aspirated. It was then buffered with $8.4 \%$ sodium bicarbonate solution. They found that pain reduction was observed in all three groups, and there was no statistically significant difference between the groups at a 3-month follow-up [40*]. They also measured the following secondary outcomes: ultrasonographic changes in tendon thickness and color Doppler activity. They reported that glucocorticoid was more effective than PRP and saline in both reducing color Doppler activity as well as reducing tendon thickness. Limitations of this study include that a local anesthetic was used in the peritendon, and this may have decreased the therapeutic effect of the PRP [35]. The authors describe that the initial primary outcome measure was going to be the PRTEE at 12 months post-procedure. However, there was significant drop-out during the study in all 3 groups, and therefore, the primary outcome was changed to 3 months post-procedure. They also explained that $60 \%$ of the subjects in the glucocorticoid group were not naïve to steroid injection treatment. It was possible that patients initially treated with glucocorticoid to good effect would not have been referred for further treatment; therefore, it was not surprising that these subjects failed to have a successful result the second time they were treated with a steroid injection. Strengths of this study include that all participants were given a standard stretching and 
strengthening protocol after the procedure [40 ${ }^{\bullet}$. A careful review of this study demonstrates the rapid early effects of corticosteroid injection over the first 6 weeks following injection, followed by a progressive decline in efficacy. This contrasts with the slow improvement slope of the PRP group. At the 3-month mark, the graphical representation of the responses demonstrates the PRP group continuing a downward slope of improvement that intersects the upward, decreasing benefit of the corticosteroid injection. This pattern (rapid benefit with regression over time) is a common finding in reviewing the literature on corticosteroid injections for tendinopathy [41].

\section{Achilles tendon}

In 2010, de Vos et al. [42] published a double-blind, randomized, placebo-controlled trial that was performed on a group of 54 patients. They used the Victorian Institute of Sports Assessment-Achilles (VISA-A) as a primary outcome measure. The authors reported that this is a validated questionnaire specifically designed for evaluating outcomes in Achilles tendinopathy. Achilles tendinopathy was treated with eccentric strengthening exercises and either a PRP injection or a saline injection. The PRP was prepared by performing a single centrifugation, and then the injectate was buffered with $8.4 \%$ sodium bicarbonate. This study showed improvement in both groups, but there was not a statistically significant difference between the saline group and the PRP group [42]. Strengths of this study include the design being double-blinded, randomized, and controlled. Additionally, all subjects were given a standard post-procedure rehabilitation protocol. The same physician performed all the injections. A significant limitation of this study was their exclusion criteria. They specifically excluded any patient who had previously completed a heavy load eccentric exercise program. Therefore, this injection was performed on patients naïve to eccentric strengthening exercises. This goes against a common recommendation that PRP treatment be performed in tendinopathy cases that are recalcitrant to an eccentric strengthening protocol. In their conclusion, the authors recommend that PRP treatment not be used in chronic midportion Achilles tendinopathy. In line with their experimental design, it may be more accurate to not recommend PRP treatment in Achilles chronic mid-portion tendinopathy in patients that are naïve to eccentric strengthening exercises. Ultrasonographic tendon evaluation of the same group of subjects showed no statistically significant differences in tendon structure or neovascularization in the PRP group compared to the saline injection group [43]. One-year follow-up of the same group of subjects did not show statistically significant differences in VISA-A score or ultrasonographic appearance of the PRP group compared to the saline injection group [44•]. It is also noteworthy that significant improvement occurred in both groups in this study, and therefore, what lead to those improvements (the needling itself, the injectate, and/or the eccentric exercise program) is not clear from this study.

\section{Patellar tendon}

In a cohort study of 20 male athletes with a mean history of 20.7 months of patellar tendon pain, a series of 3 PRP injections to treat patellar tendinopathy was performed to assess the efficacy of this treatment for this condition [45]. They described that the primary purpose of the study was to explore PRP for the treatment of chronic patellar tendinosis, and to specifically assess adverse events of subjects before and after treatment. The secondary purpose was to measure the results of the treatment. The blood went through two serial centrifugations, and then the PRP was aspirated. Before each injection, the PRP was mixed with calcium chloride to activate the platelets. After the first injection, the patients were allowed to use non-steroidal anti-inflammatory drugs (NSAIDS) for somewhere between $24 \mathrm{~h}$ up until the second injection. The exact amount of time for which NSAIDS were permitted was not clear in the paper. After the second injection, stretching and mild activities were suggested. After the third injection, participants were encouraged to begin a strengthening program. After 1 month they were advised to return to activities as tolerated. The outcome measures used were Tegner, EQ-VAS, and SF-36. The mean clinically significant difference was set at 15 points. At 6 months' followup, all parameters of the SF-36 demonstrated both statistically and clinically significant improvement. The EQVAS was also reported to demonstrate statistically significant improvement. The Tegner score assessment was described to show a statistically significant improvement in the patients. They further report that most of the men returned to the sport with a lower score than their score prior to their injury. However, the score of most of these men was not statistically significantly different than their score prior to their injuries. The strength of this study as reported was that they did achieve their purpose, which was to evaluate the safety of their protocol. There were several limitations to this study. They described the purpose as primarily looking for adverse events, and therefore the results of the treatment were only a secondary purpose. This was a cohort study, and therefore no control group was present. They allowed the patients to use NSAIDS for an inexact period of time for pain control between the first and second injections, which could have inhibited some of the therapeutic effect. The patients had tried a variety of 
treatments prior to these injections, but none of these prior treatments was common to all the participants. This protocol also involved a series of three PRP injections. These injections can be several hundred dollars each, and the cost of three injections may be prohibitive to a large portion of patients [45].

Filardo et al. [46] performed a cohort control study in order to assess the therapeutic effect of three PRP injections in patients with chronic tendinopathy. They compared a group of 15 patients who received a series of 3 PRP injections for patellar tendinopathy to a group of 16 patients treated primarily with physical therapy. The blood was put through two serial centrifugations, and then the PRP was aspirated. The PRP was mixed with calcium chloride prior to injection in order to activate the platelets. They reported that Tegner, EQ-VAS, and pain scale were used as outcome measures. The authors did not specifically explain how they chose to use these particular outcome measures. At 6-month follow-up, there was not a difference in EQ-VAS or pain scale between the two groups. The authors did report that the PRP group did achieve a greater improvement in sport activity level relative to the control group. This study had several limitations. The control group had not had a course of physical therapy prior to their intervention; thus, the two groups had a different preintervention treatment regimen. Furthermore, the study was neither blinded nor randomized [46].

De Almeida et al. [47] discussed that sports injuries have heterogeneity of lesions, which makes it difficult to compare efficacy of treatments in prospective randomized trials. They also reviewed basic science literature that showed PRP improved the mechanical properties of a rabbit's patellar tendon after resection of its central portion [48]. Therefore, they used the patellar tendon harvest site as an experimental model to assess the effect of PRP on patellar tendon healing in humans in a prospective, randomized, evaluator-blinded study. The primary outcome was magnetic resonance imaging (MRI) assessment of patellar tendon harvest site healing. Secondary outcomes were functional and clinical evaluations of ACL reconstruction with a patellar tendon graft to examine whether adding PRP to the harvest site affects the clinical and functional outcomes of the procedure. PRP was obtained by using a cell separator with a specific kit for platelet apheresis in the operating room simultaneously with ACL reconstruction. Calcium chloride was added to one of the vials of PRP to activate the platelets. With regards to the primary outcome, PRP treatment to the patellar tendon harvest site for ACL graft harvesting showed a smaller tendon gap at 6 months relative to a control group [47]. On the Tegner questionnaire, both groups had worse results. When comparing the questionnaire scores of the two groups, there was no statistically significant difference.
Notably in this study, due to the method of obtaining PRP, $30-50 \mathrm{ml}$ of PRP was obtained for each patient. There were several strengths of this study. A single surgeon performed all the procedures. There was a standard defect in the central portion of the patellar tendon, therefore the authors could compare patellar tendon harvest site healing after a standardized and well-established procedure. A single musculoskeletal trained radiologist performed all the blinded MRI evaluations. All subjects followed the same rehabilitation protocol. Limitations of this study included that post-procedure analgesic medications included ketoprofen, and this may have blunted the therapeutic efficacy of the PRP [35]. Additionally, the surgeon performing the procedures was not blinded to the two treatment groups.

A randomized controlled trial of 46 athletes compared the treatment of patellar tendinopathy with either 2 PRP injections or 3 sessions of focused extra-corporeal shock wave (ECSW) therapy [49॰]. The PRP was obtained by performing a single centrifugation of whole blood, and the PRP was then collected and stored until ready for injection. The outcome measures were the Victorian Institute of Sports Assessment-Patella (VISA-P) questionnaire, the pain visual analog scale (VAS) during five single-legged squats on the affected knee, and a modified Blazina scale. The authors report VISA-P is the only published clinical scale validated for patellar tendinopathy. Using the VISA-P scale, there was statistically significant improvement in the PRP group relative to the ECSW group at 6 months (PRPI86.7 [SD $=14.2], \mathrm{ECSW}-73.7$ [19.9], $P=0.014)$ and at 12 months (PRP-91.3 [SD = 9.9], ECSW-77.6 [19.9], $P=0.026$ ) [49॰]. At 6- and 12-month follow-ups, there was a greater improvement in VAS scores after 5 single-legged squats in the PRP group compared to the ECSW group. At a 12-month follow-up, there was also a greater improvement in the modified Blazina scale in the PRP group relative to the ECSW group. Strengths of this study include that the same physician performed all the procedures. They also avoided the use of anesthetics in both treatment groups. Limitations of this study include that the patients were not blinded to the intervention, and it was not placebo-controlled. It is possible that awareness of the treatment modality may have had some effect on the patients' perception of their response to the treatment.

In the multi-center, retrospective study by Mautner et al. [50], the patellar tendon was found to be the most difficult to treat, with greater than $50 \%$ or more improvement noted in only $59 \%$ of patients treated for chronic patellar tendinopathy.

\section{Rotator cuff tendinopathy}

Bergeson et al. [51] performed an observational cohort study on 16 patients with at-risk arthroscopic rotator cuff 
repairs to determine the effect of platelet-rich fibrin matrix (PRFM) augmentation on healing rates and functional outcome scores. They used an algorithm to determine patients with a rotator cuff tear that was at risk for a re-tear. They used a retrospective control group of 21 patients that had been operated on by the same group of surgeons performing the PRP-augmented repair. The PRFM was created by performing serial centrifugations on autologous whole blood. The second centrifugation was done with calcium chloride added, in order to initiate the fibrin clotting cascade. Several functional outcome scales were used to assess functional improvement in the two groups. These included the American Shoulder and Elbow Surgeons system (ASES), the Constant system, the University of California at Los Angeles (UCLA) system, the Western Ontario Rotator Cuff Index (WORC), and Single Assessment Numeric Evaluation (SANE). There was no statistically detectable difference between the PRFM group and the retrospective control group with respect to postoperative functional scores. MRIs were also performed at 1 year post-surgery in all patients except three. The percentage of re-tears in the PRFM group was $56 \%$, and in the historical control group was $38 \%$. This difference reached statistical significance $(P=0.024)$. Weaknesses of this study include no randomization and the inherent selection bias due to the use of an historical control group. There was heterogeneity of repair technique, which may have also influenced the results. The mean follow-up time for the PRFM group functional scores was 13 months, and the mean follow-up time for the historical control group functional scores was 27 months, which also may have introduced bias.

Rodeo et al. [52] performed a randomized trial on 79 patients undergoing surgical repair of full-thickness rotator cuff tears. Forty patients received the experimental treatment, which was surgical repair augmented with PRFM, and 39 patients received surgical repair without augmentation. The primary outcome measure was ultrasonographic evidence of postoperative tendon healing at 6 and 12 weeks. Secondary outcomes included standardized shoulder outcome scales and strength measurements. The standard postoperative rehabilitation regimen was prescribed in all patients. The authors report that the PRFM was made intra-operatively by obtaining peripheral venous blood at the start of the case. They report that they used a second centrifugation and added calcium chloride during this step in order to activate the fibrin-clotting cascade. At 6 weeks, 30 of $36(83 \%)$ rotator cuff tendons were intact in the control group, and 28 of $34(82.4 \%)$ rotator cuff tendons were intact in the PRFM group $(P=0.913)$. At 12 weeks, 25 of $31(80.6 \%)$ rotator cuff tendons were intact in the control group, compared with 24 of 36 $(66.7 \%)$ in the PRFM group $(P=0.198)$. Strengths of this study included that the evaluating sonographer was blinded to which treatment each patient received. Weaknesses of this study include that the surgeons performing the procedure were not blinded to which patients were receiving the PRFM augmentation. The follow-up points were also relatively soon after the surgery.

In a prospective cohort study, 42 patients had arthroscopic repair of full-thickness rotator cuff tears, either with or without PRP to augment the surgery [53]. The patients made the decision whether or not they wanted to have PRP used. The PRP was obtained by using a plateletpheresis system with a leukoreduction set. In order to form a gel, calcium gluconate was added to the PRP. After the medial row of sutures were threaded, the PRP gel was applied. The outcome assessments included pain, ROM, strength, and functional scores and overall satisfaction at periodic intervals up to 16 months. Several functional scales were used: American Shoulder and Elbow Surgeons system, the Constant system, the University of California at Los Angeles (UCLA) system, the Disabilities of the Arm, Shoulder and Hand (DASH) system, the Simple Shoulder test, and the SPADI. The majority of functional scores showed no significant difference at any of the follow-up time periods, with the exception of the 3-month follow-up. At this point, the ASES score, the Constant score, and the SPADI score showed increased functional improvement in the control group. Additionally, the authors commented that the PRP group had a lower re-tear rate-26.7\% compared to $41.2 \%$; however, they report that this was not statistically significant. Strengths of this study include the standardization of the PRP gel. The authors report that they focused on two factors to obtain meaningful resultsstandardization of PRP production and the reproducible application of PRP. They obtained this by using a plateletpheresis system instead of a desktop system. They also used a gel because they felt that an injection might be diluted or washed out during an arthroscopic procedure due to the nature of the procedure. All subjects had the same postoperative protocol-shoulders were immobilized for 4-6 weeks using an abduction brace. They gradually progressed to short arc range of motion, then passive range of motion and then active assisted range of motion. Patients returned to sports after 6-9 months according to individual recovery. The weaknesses of this study include lack of randomization, a larger proportion of large and massive tears in the PRP group, and an arbitrary volume, concentration, and activation level for the PRP.

Kesikburun et al. [54•] performed a randomized controlled trial on 40 patients with rotator cuff tendinosis or partial thickness rotator cuff tears. The patients received either a single PRP injection or a single saline injection. The PRP was obtained by performing a single centrifugation on whole blood, and then separating out the PRP. The primary outcome measure was the Western Ontario Rotator 
Cuff Index (WORC). The authors reported the WORC is a valid and reliable disease-specific, quality of life selfassessment measurement tool for rotator cuff disease. The authors attempted to detect a clinically relevant difference of $17 \%$ in the WORC score between the 2 groups. This study demonstrated no statistically significant differences in the WORC score between the two treatment groups in follow-ups at weeks 3, 6, 12, and 24, or at one year [54•]. Strengths of this study include that it was double-blinded, and the clinician performing the injection, the patient, and the evaluator were blinded as to which injection the patient received. A single clinician performed all the injections. All patients underwent the same post-procedure rehabilitation protocol. The patients were told to avoid NSAIDS after the procedure. Limitations of this study include that the rotator cuff was anesthetized with lidocaine prior to either injection, which may decrease the therapeutic effect of the PRP [35].

Rha et al. [55•] performed a prospective randomized, double-blind, controlled study in 39 patients with rotator cuff tendinosis or partial-thickness tears. They compared the effects of two serial PRP injections that were performed 4 weeks apart to two serial dry needling procedures performed 4 weeks apart. The PRP was obtained by performing two serial centrifugations on autologous whole blood. SPADI was the main outcome measurement. Follow-up evaluations were performed at several intervals up to 6 months after the second injection. Acetaminophen and hydrocodone were used for post-procedure pain control. After the second injection, they found that at 2-week follow-up, at 3-month follow-up, and at 6-month follow-up, the PRP treated group had statistically detectable improvement relative to the dry needling group, using the SPADI [48]. They did not specifically address the clinical significance of the statistically detectable improvement in the overall SPADI score. However, they did also perform a separate analysis of the total pain score subset of SPADI score between the two groups, and the total disability score subset of SPADI score between the two groups. The authors note that there was no statistically detectable difference between the two groups at any time point when comparing the total pain score subset and total disability score subset. Strengths of the study include that the patients were blinded as to which treatment they received. Weaknesses of this study include that the physician performing the procedure was not blinded as to which injectate he was using. Due to this fact, the study was not technically double-blind, because this presents the possibility of bias from the proceduralist, which may have affected the outcomes. There was also a $25 \%$ drop-out rate in this study by the end of the 6-month follow-up period, which may bias the results. The authors do not describe a specific standardized rehabilitation protocol for all subjects in the methods section.

\section{Multi-tendon tendinopathy studies}

In a retrospective cross-sectional survey, Mautner et al. [50] assessed the results of 325 patients who received ultrasound-guided PRP injections for tendinopathy that was refractory to conventional management. Only 180 patients that were contacted answered the survey. The primary outcome measurement was the perceived improvement in symptoms at least 6 months after the PRP injection(s). This perception was quantified using the following Likert scale: "Not at all," "Slightly," "Moderately," "Mostly," and "Completely". The primary outcome measurement (improvement in symptoms) was analyzed by calculating a global average for all tendons, average improvement for each of the most commonly treated tendon groups, and average improvement according to the number of injections received. Secondary outcome measurements were the following: perceived change in VAS before and after the procedure (from 0 for no pain to 10 for worst pain), functional pain after the procedure using the Nirschl Pain Phase Scale for overuse injuries, and overall satisfaction with the PRP procedure (quantified with the following Likert scale: "Completely Dissatisfied," "Mostly Dissatisfied," "Somewhat Dissatisfied," "No Difference," "Somewhat Satisfied," "Mostly Satisfied," and "Completely Satisfied"). $82 \%$ of patients that responded at 1 year or greater post-procedure recorded a moderate-tocomplete resolution of symptoms ( $\geq 50 \%$ improvement). The three most common tendons treated were the insertion of the common extensor tendon at the lateral epicondyle, the Achilles, and the patellar tendons. $93 \%$ of patients who received a lateral epicondyle injection, $100 \%$ of patients who received an Achilles injection, and $59 \%$ of patients who received a patellar tendon injection reported moderate to complete resolution of symptoms ( $\geq 50 \%$ improvement). Over $80 \%$ of patients that received an injection in the rotator cuff, hamstring, gluteus medius or the common flexor tendon at the medial epicondyle reported $\geq 50 \%$ improvement. $60 \%$ of patients received only one injection; $30 \%$ received two injections, and $10 \%$ received three or more injections. There was an average reduction in VAS of $74 \%$ noted. Strengths of this study include that it represents the largest database of patients treated with PRP for tendinopathy that has been published at the time of its publication. Weaknesses of this study include that all the patients had not uniformly attempted and failed an eccentric strengthening therapeutic exercise program prior to having the PRP procedure performed. Rather, the authors state that the inclusion criteria was a diagnosis of tendinopathy for $>6$ months that had not resolved with conventional treatments, including oral medications, physiotherapeutic modalities, and eccentric exercises (those that involve slow, controlled lengthening of the 
muscle/tendon unit), among others. This is further accentuated by the fact that the authors document that the patients must have completed a rehabilitation program that included eccentric exercises no earlier than 4 weeks after the procedure. This description makes it difficult to determine what portion of improvement was from the PRP injection versus from the eccentric exercise rehabilitation that they completed after the injection. This is especially true given that they had not necessarily completed an eccentric exercise program prior to the PRP injection, based on the inclusion criteria described. Only $55 \%$ of the patients that were contacted responded to the survey, which introduces selection bias as a possible confounder. The study collected retrospective data, which results in recall bias, does not control for confounding factors, and limits the type of questions that can be asked.

In a two-part study, Finoff et al. examined 41 subjects who first received a single ultrasound-guided percutaneous needle tenotomy and PRP injection. In the second part of the study, a diagnostic musculoskeletal ultrasound examination was performed on the tendon that was treated with PRP [56]. The PRP used for the first part of the study was obtained by processing autologous whole blood in one of two different types of platelet concentrating devices. The PRP was buffered with $8.4 \%$ sodium bicarbonate prior to being injected into the tendinopathic lesion. After the injection, all patients were given the same progressive stepwise rehabilitation protocol. The outcomes of primary interest included clinical outcomes (pain severity [including average, worst, and best], functional limitations, and satisfaction) and tendon morphology (thickness, length of tendinopathy, echotexture, and extent of neovascularization). They report that a 3-point or greater improvement in pain or function was considered clinically significant, because a Cochrane review suggested that the placebo effect on pain was approximately $24 \mathrm{~mm}(24 \%)$ on a 100-mm VAS for pain [57].

They found that the mean time to maximum improvement was 4 months. The mean functional improvement was $68 \%$, and the mean "worst-pain" improvement was $58 \%$. There was an $84 \%$ improvement in echotexture, and $83 \%$ of the patients were satisfied with their outcome [56]. Strengths of the study include that all subjects completed the standardized post-procedure rehabilitation protocol. Strict inclusion and exclusion criteria were used when selecting patients for the procedure, which reduced the heterogeneity of the patient population. Only two providers performed the procedures, thus reducing interoperator variability of the procedure. Weaknesses of this study include that all the patients had not definitively performed an eccentric strengthening protocol, but rather eccentric strengthening was reported to be performed "where applicable". $2 \mathrm{ml}$ of $1 \%$ lidocaine was used to anesthetize the tendon prior to the tenotomy and PRP injection, which may have blunted the therapeutic effect [35]. There was not a control group. It was also a retrospective study, which can be subject to a variety of biases.

\section{Evidence regarding use of PRP for ligament injuries}

\section{Anterior cruciate ligament (ACL)}

There is little research regarding the role of PRP for most ligament injuries, including the ACL. Murray et al. [58] showed that placement of a collagen-PRP scaffold in a central ACL defect in pigs, at the time of surgical repair, was able to promote ACL healing both histologically and biomechanically at a 4-week follow-up.

In a Level 1 study Silva, KSSTA 2009 noted that PRP did not accelerate healing of autologous HS ACL reconstruction as assessed by MRI [59].

In a Level III, case-control study of anterior cruciate ligament reconstruction, there was noted improved ligamentization of tendon grafts treated with an endogenous preparation rich in growth factors [60].

\section{Ulnar collateral injury of the elbow}

In a prospective cohort study, 34 athletes with MRI-confirmed partial tears of the ulnar collateral ligament were treated with a single PRP injection under ultrasound guidance [61]. The investigators found that 30 of 34 athletes $(88 \%)$ had returned to the same level of play without any complaints with an average follow-up of 70 weeks. The average time to return to play was 12 weeks (range 10-15 weeks). The average KJOC score improved from 46 to $93(P<0.0001)$. The average DASH score improved from 21 to $1(P<0.0001)$. DASH questionnaire improved from 69 to $3(P<0.0001)$. Medial elbow joint space opening with valgus stress decreased from 28 to $20 \mathrm{~mm}$ at final follow-up $(P<0.0001)$. The difference in medial elbow joint space opening (stressed vs. non-stressed) decreased from 7 to $2.5 \mathrm{~mm}$ at final follow-up $(P<0.0001)$. One player had persistent UCL insufficiency and required ligament reconstruction 31 weeks after injection [61].

\section{Evidence regarding use of PRP for joint and cartilage damage}

Knee joint

In an uncontrolled, prospective preliminary study, 14 patients with primary or secondary knee OA received intra- 
articular PRP injections at 4-week intervals for three total injections [62]. Several outcome measures were used, including the Brittberg-Peterson Visual Analog Pain, Activities, and Expectations Score, which included a $10-\mathrm{mm}$ VAS with resting, walking and with the knee in a bent position; and the five subscale Knee Injury and Osteoarthritis Outcome Score. The Brittberg-Peterson scale showed statistically significant overall improvement with respect to resting pain, moving pain, and bent knee pain $(P<0.05)$. At one-year follow-up, eight of the patients indicated that they achieved their individual goal with the injection; three patients indicated the pain was the same, and two patients indicated the knee pain had worsened.

A cohort of 100 patients with degenerative knee cartilage lesions received a series of 3 PRP injections administered at 21-day intervals [63]. The outcome measures used were the International Knee Documentation Committee (IKDC) and EuroQol VAS (EQ VAS). There was a statistically significant improvement in both outcome measures from baseline to 6-month and baseline to 12-month follow-up $(P<0.0005)$. The results significantly worsened from 6 to 12 months, although the results at 12 months were still significantly higher with respect to baseline $(P<0.0005)$. There was a statistically significant improvement in the IKDC score at 6-month follow-up. Poorer outcomes were associated with older age, more severe grade of osteoarthritis, and higher body mass index.

In a prospective cohort study, twenty patients with early knee osteoarthritis received a single PRP injection [64]. Fifteen of the patients had clinical assessments at baseline, 1 week, and 1, 3, 6 and 12 months. They also had MRIs at 1 year. VAS scores and Western Ontario McMaster Universities Arthritis Index (WOMAC) scores were used as outcome measures. There was a statistically significant $56.2 \%(P<0.001)$ reduction in mean baseline VAS pain scores at 6 months, and a $58.9 \%$ reduction in mean VAS pain scores $(P=0.001)$ at 1 year [55]. The overall scores also improved significantly at 6 months by $45.1 \%$ $(P=0.003)$ and at 12 months by $56.2 \%(P=0.002)$.

In a prospective cohort study, 65 patients with knee OA were treated with a single PRP intra-articular knee injection [65]. VAS and IKDC scores were used as outcome measures. Overall, the average VAS score improved from 7.4 before the procedure to $5.0,4.5$, and 4.2 for 1,3 , and 6 months after procedure, respectively. However, the clinical symptoms tended to deteriorate to 4.7 and 5.0 for 9 months and 1 year, respectively. On average, patients reported relapse of knee pain at an average of 8.8 months after the procedure. The mean IKDC score changed from 54.1 before the procedure to 53.9 at 1 month post-procedure, 61.6 at 6 months post-procedure, and 50.3 at 1 year post-procedure. There was a shortened time to the re-onset of pain according to $\mathrm{KL}$ grade $(P=0.037)$. If $\mathrm{PFJ}$ degeneration occurred, the pain returned at 7.9 months on average; if FPJ was not present, pain returned at an average of 10.2 months, and this was reported to be statistically significant $(P=0.038)$. There was also the suggestion that at greater age, the clinical effect was attenuated.

Gobbi et al. [66] examined a 50-patient case series (25 patients with a prior operative intervention for a cartilage lesion and 25 patients with surgically naïve knees) who were given two intra-articular knee PRP injections to treat knee OA. The primary outcome measure was the IKDC. All patients showed significant improvement in all scores at 6 and 12 months $(P<0.01)$ and returned to previous activities, including recreational sports.

In a prospective comparative study, 150 patients were divided to receive either 3 autologous PRP injections, 3 high molecular weight HA (HWHA) injections, or 3 low molecular weight HA injections (LWHA) [67]. All injections were given at 2-week intervals. IKDC and EQ VAS scales were used for outcome measures. A statistically significant improvement in all clinical scores from baseline evaluation to the 2- and 6-month follow-up evaluations was observed in all treatment groups. There was a higher number of satisfied patients in the PRP group (82\% in PRP group, $64 \%$ in LWHA group and $66 \%$ in the HWHA group; $P=0.04$ ). The analysis at the 6 -month follow-up, the primary outcome of the study, showed better IKDC results in the PRP group compared with the LW HA group $(P=0.003)$, as well as compared with patients treated with HW HA $(P=0.005)$. EQ VAS also showed greater improvement in the PRP group compared to the other two groups (PRP vs. LWHA, $P=0.001$; PRP vs. HWHA, $P=0.002$ ). There were overall worst results in patients aged over 50 years: at 6 months of follow-up, IKDC evaluation showed lower scores in older patients in the PRP group $(P=0.004)$, as well as in the LW HA group $(P=0.003)$ and HW HA group $(P=0.003)$. More severe degeneration was also associated with worse outcomes in all three groups.

Spaková et al. [68] performed a prospective cohort/ control study in which 120 patients received either three PRP intra-articular knee injections or three hyaluronic acid (HA) injections [68]. Outcome measures included the Western Ontario and McMaster Universities Osteoarthritis Index (WOMAC) and the 11-point pain intensity numeric rating scale (NRS). At 3- and 6-month follow-ups, the PRP group showed statistically significant improvement compared to the HA group in both outcome measures $(P<0.01$ in both outcome measures). In the PRP group, the mean score of the WOMAC improved from 38.76 $(\mathrm{SD}=16.50)$ at baseline to $14.35 \quad(\mathrm{SD}=14.18)$ at 3-month follow-up and to $18.85(\mathrm{SD}=14.09)$ at 6-month follow-up. The mean score of the 11-point pain intensity 
NRS was $5.27(\mathrm{SD}=1.87)$ at baseline, $2.06(\mathrm{SD}=2.02)$ at 3 -month follow-up, and $2.69(\mathrm{SD}=1.86)$ at 6 -month follow-up. In the HA group, the WOMAC improved from $43.21(\mathrm{SD}=13.70)$ to $26.17(\mathrm{SD}=17.47)$ at 3 -month follow-up and to $30.90(\mathrm{SD}=16.57)$ at 6 -month follow up. The mean score of the NRS was $6.02(\mathrm{SD}=1.77)$ at baseline, $3.98(\mathrm{SD}=2.27)$ at 3-month follow-up, and 4.3 $(\mathrm{SD}=2.07)$ at 6 -month follow-up.

In an observational retrospective cohort/control study, Sánchez et al. [69] examined 30 patients who received 3 weekly injections of an autologous preparation rich in growth factors, and 30 patients received 3 weekly injections of HA. WOMAC was measured at baseline and at 5 -week follow-up. The observed success rates by week 5 for the pain subscale reached $33.4 \%$ for the autologous preparations rich in growth factors (PRGF) group and $10 \%$ for the hyaluronan group $(P=0.004)$.

A randomized controlled trial of 120 patients compared two groups who received either 4 weekly PRP injections or 4 weekly HA injections [70•]. The WOMAC score was the primary outcome measure. At week 24, the PRP group had continuous improvement, whereas the subjects treated with HA showed a sharp worsening. The mean WOMAC score was 36.5 in the ACP group (range 5-76; $\mathrm{SD}=617.9$ ) and 65.1 in the HA group (range $41-82$; $\mathrm{SD}=610.6)(P<0.001)$. The PRP group also had better functional scores at 4 and 12 weeks follow-up compared to the HA group $(P<0.001$ and $P<0.001$, respectively). A statistically significant difference between grade III gonarthrosis treated with ACP and that treated with HA was observed at week 12 as well as at week 24 , with a noticeable improvement that was greater in the patients treated with ACP $(P<0.001)$. Other than the historical use of viscosupplement injections in a series of 3 weekly injections, the rationale for performing PRP injections in either 3 or 4 weekly injections is not substantiated by basic science or clinical practice.

Hip joint

Sánchez et al. [71] examined 40 patients with severe unilateral hip OA who each received a series of 3 intra-articular hip PRP injections. The primary end point was meaningful pain relief, which was described as a reduction in pain intensity of at least $30 \%$ from baseline levels as evaluated by the WOMAC subscale at 6 months post-treatment. There was a significant reduction in the WOMAC pain scores over the 6 to 7 -week $(W=438 P=0.00047)$ and 6-month periods $(W=516, P=0.00607)$.

Ankle joint

Mei-Dan et al. [72] assessed the efficacy of using PRP for non-invasive management of osteochondral lesions (OCL) of the talardome. They performed a randomized controlled trial on 30 OCLs. The patients were randomized to receive three consecutive injections of either HA or PRP. The follow-up points were at 4,12 , and 28 weeks. The primary outcome measures were VAS scale and Ankle Hindfoot Scale (AHFS). Both groups were reported to have significant improvement at all follow-up points compared to baseline. The AHFS score improved from 66 and 68 to 78 and 92 in groups 1 and 2, respectively, from baseline to week $28(P<0.0001)$, favoring PRP $(P<0.05)$. Mean VAS scores $(1=$ asymptomatic, $10=$ severe symptoms $)$ decreased for pain (group 1: 5.6 to 3.1; group $2: 4.1$ to 0.9 ), stiffness (group 1: 5.1 to 2.9; group 2: 5.0 to 0.8 ), and function (group 1: 5.8 to 3.5; group 2: 4.7 to 0.8 ) from baseline to week $28(P<0.0001)$, favoring PRP $(P<0.05$ for stiffness, $P<0.01$ for function, $P<0.05$ for pain). Subjective global function scores, reported on a scale from 0 to 100 (with 100 representing healthy, preinjury function) improved from 56 and 58 at baseline to 73 and 91 by week 28 for groups 1 and 2 , respectively $(P<0.01$ in favor of PRP). The authors concluded that the group receiving the PRP treatment had a significantly greater improvement compared to the group receiving the HA treatment.

\section{Muscle injury}

At the Second World Congress on Regenerative Medicine in 2005, a poster was presented describing the treatment of 20 professional athletes with muscle injury [73]. Based on the severity of the injury, the athletes were treated with 1-3 injections of PRGF. Swelling and pain were reduced, and functional capabilities were fully restored in half the expected recovery time. Ultrasonographic images showed full regenerated muscle tissue after treatment, and fibrosis did not appear in any of the treated cases. This differs from the normal pattern of muscle recovery following a muscle strain, which generally includes disruption of the normal architecture of the muscle fibers followed by muscle regeneration; this is often associated with muscle fibrosis. Depending on the severity of the injury, recovery can take as long as $4-6$ weeks, with return to sport participation taking even longer.

\section{Conclusions}

There is evolving evidence for the efficacy of PRP for a variety of musculoskeletal conditions. The best studies have been performed in chronic tendinopathy, with positive results noted for chronic lateral epicondylosis. There has been mixed evidence in other tendons, in particular the Achilles tendon, where in a randomized, controlled study PRP was found to be no better than a saline injection. PRP 
does not appear to provide additive benefit in conjunction with rotator cuff repair. There is good and increasing evidence for the efficacy of PRP (in reducing pain and improving daily function) in osteoarthritis of the knee and limited but increasing evidence for other joints. PRP injections appear to have superior efficacy to HA injections. PRP use appears to be effective in the treatment of partial tears of ulnar collateral injury of the elbow in baseball pitchers, but this is based on a single study. Its efficacy for other ligament injuries and muscle tears has not been proven. There are various factors that must be addressed when considering the use of PRP, and when reviewing prior research of $\mathrm{PRP}$, which include platelet concentration, leukocyte count, $\mathrm{pH}$ of the injected substance, use of activators, the total number of injections given, the method of delivery, and perhaps most importantly, the type of post-procedure rehabilitation protocol.

\section{Compliance with Ethics Guidelines}

Conflict of Interest G. A. Malanga declares no conflicts of interest. M. Goldin declares no conflicts of interest.

Human and Animal Rights and Informed Consent This article does not contain any studies with human or animal subjects performed by any of the authors.

\section{References}

Papers of particular interest, published recently, have been highlighted as:

- Of importance

1. Marx RE. Platelet-rich plasma (PRP): what is PRP and what is not PRP? Implant Dent. 2001;10(4):225-8.

2. Weibrich G, Hansen T, Kleis W, et al. Effect of platelet concentration in platelet-rich plasma on peri-implant bone regeneration. Bone. 2004;34(4):665-71.

3. Giusti I, Rughetti A, D'Ascenzo S, et al. Identification of an optimal concentration of platelet gel for promoting angiogenesis in human endothelial cells. Transfusion. 2009;49(4):771-8.

4. Kevy SV, Jacobson MS. Comparison of methods for point of care preparation of autologous platelet gel. J Extra Corpor Technol. 2004;36(1):28-35.

5. Ferrari M, Zia S, Valbonesi M, et al. A new technique for hemodilution, preparation of autologous platelet-rich plasma and intraoperative blood salvage in cardiac surgery. Int $\mathrm{J}$ Artif Organs. 1987;10(1):47-50.

6. DelRossi AJ, Cernaianu AC, Vertrees RA, et al. Platelet-rich plasma reduces postoperative blood loss after cardiopulmonary bypass. J Thorac Cardiovasc Surg. 1990;100(2):281-6.

7. Marx RE, Carlson ER, Eichstaedt RM, et al. Platelet-rich plasma: growth factor enhancement for bone grafts. Oral Surg Oral Med Oral Pathol Oral Radiol Endod. 1998;85(6):638-46.

8. Anitua E, Andia I, Ardanza B, et al. Autologous platelets as a source of proteins for healing and tissue regeneration. Thromb Haemost. 2004;91(1):4-15.
9. Duffy FJ, Seiler JG, Gelberman RH, et al. Growth factors and canine flexor tendon healing: initial studies in uninjured and repair models. J Hand Surg Am. 1995;20(4):645-9.

10. Klein MB, Yalamanchi N, Pham H, et al. Flexor tendon healing in vitro: effects of TGF-beta on tendon cell collagen production. J Hand Surg Am. 2002;27(4):615-20.

11. Marui T, Niyibizi C, Georgescu HI, et al. Effect of growth factors on matrix synthesis by ligament fibroblasts. J Orthop Res. 1997;15(1):18-23.

12. Koch RM, Roche NS, Parks WT, et al. Incisional wound healing in transforming growth factor-beta1 null mice. Wound Repair Regen. 2000;8(3):179-91.

13. Nathan C. Points of control in inflammation. Nature. 2002;420(6917):846-52.

14. Blaney Davidson EN, Vitters EL, van der Kraan PM, et al. Expression of transforming growth factor-beta (TGFbeta) and the TGFbeta signalling molecule SMAD-2P in spontaneous and instability-induced osteoarthritis: role in cartilage degradation, chondrogenesis and osteophyte formation. Ann Rheum Dis. 2006;65(11):1414-21.

15. Verdier M-P, Seité S, Guntzer K, et al. Immunohistochemical analysis of transforming growth factor beta isoforms and their receptors in human cartilage from normal and osteoarthritic femoral heads. Rheumatol Int. 2005;25(2):118-24.

16. Boyer MI, Watson JT, Lou J, et al. Quantitative variation in vascular endothelial growth factor mRNA expression during early flexor tendon healing: an investigation in a canine model. J Orthop Res. 2001;19(5):869-72.

17. Buckley A, Davidson JM, Kamerath CD, et al. Sustained release of epidermal growth factor accelerates wound repair. Proc Natl Acad Sci USA. 1985;82(21):7340-4.

18. Abrahamsson SO. Similar effects of recombinant human insulinlike growth factor-I and II on cellular activities in flexor tendons of young rabbits: experimental studies in vitro. J Orthop Res. 1997; 15(2):256-62.

19. Phornphutkul $\mathrm{C}, \mathrm{Wu} \mathrm{K}-\mathrm{Y}$, Yang $\mathrm{X}$, et al. Insulin-like growth factor-I signaling is modified during chondrocyte differentiation. J Endocrinol. 2004;183(3):477-86.

20. Mautner K, Malanga G, Colberg R. Optimization of ingredients, procedures and rehabilitation for platelet-rich plasma injections for chronic tendinopathy. Pain Manag. 2011;1(6):523-32.

21. Dragoo JL, Braun HJ, Durham JL, et al. Comparison of the acute inflammatory response of two commercial platelet-rich plasma systems in healthy rabbit tendons. Am J Sports Med. 2012;40(6):1274-81.

22. McCarrel TM, Minas T, Fortier LA. Optimization of leukocyte concentration in platelet-rich plasma for the treatment of tendinopathy. J Bone Joint Surg Am. 2012;94(19):e143(1-8).

23. De Mos M, van der Windt AE, Jahr H, et al. Can platelet-rich plasma enhance tendon repair? A cell culture study. Am J Sports Med. 2008;36(6):1171-8.

24. Wang X, Qiu Y, Triffitt J, et al. Proliferation and differentiation of human tenocytes in response to platelet-rich plasma: an in vitro and in vivo study. J Orthop Res. 2012;30(6):982-90.

25. Bosch G, van Schie HTM, de Groot MW, et al. Effects of platelet-rich plasma on the quality of repair of mechanically induced core lesions in equine superficial digital flexor tendons: a placebo-controlled experimental study. J Orthop Res. 2010;28(2):211-7.

26. Zhang J, Wang JH-C. Platelet-rich plasma releasate promotes differentiation of tendon stem cells into active tenocytes. Am J Sports Med. 2010;38(12):2477-86.

27. Aspenberg $\mathrm{P}$, Virchenko O. Platelet concentrate injection improves Achilles tendon repair in rats. Acta Orthop Scand. 2004;75(1):93-9. 
28. Sadoghi P, Lohberger B, Aigner B, et al. Effect of platelet-rich plasma on the biologic activity of the human rotator-cuff fibroblasts: a controlled in vitro study. J Orthop Res. 2013;31(8): 1249-53.

29. Ishida K, Kuroda R, Miwa M, et al. The regenerative effects of platelet-rich plasma on meniscal cells in vitro and its in vivo application with biodegradable gelatin hydrogel. Tissue Eng. 2007;13(5):1103-12.

30. Sun Y, Feng Y, Zhang CQ, et al. The regenerative effect of platelet-rich plasma on healing in large osteochondral defects. Int Orthop. 2010;34(4):589-97.

31. Van Buul GM, Koevoet WLM, Kops N, et al. Platelet-rich plasma releasate inhibits inflammatory processes in osteoarthritic chondrocytes. Am J Sports Med. 2011;39(11):2362-70.

32. Akeda K, An HS, Okuma M, et al. Platelet-rich plasma stimulates porcine articular chondrocyte proliferation and matrix biosynthesis. Osteoarthr Cartil. 2006;14(12):1272-80.

33. Wright-Carpenter T, Opolon P, Appell HJ, et al. Treatment of muscle injuries by local administration of autologous conditioned serum: animal experiments using a muscle contusion model. Int $\mathbf{J}$ Sports Med. 2004;25(8):582-7.

34. Mishra A, Pavelko T. Treatment of chronic elbow tendinosis with buffered platelet-rich plasma. Am J Sports Med. 2006;34(11): 1774-8.

35. Carofino B, Chowaniec DM, McCarthy MB, et al. Corticosteroids and local anesthetics decrease positive effects of platelet-rich plasma: an in vitro study on human tendon cells. Arthroscopy. 2012;28(5):711-9.

36. Peerbooms JC, Sluimer J, Bruijn DJ, et al. Positive effect of an autologous platelet concentrate in lateral epicondylitis in a double-blind, randomized, controlled trial: platelet-rich plasma versus corticosteroid injection with a 1-year follow-up. Am J Sports Med. 2010;38(2):255-62.

37. - Gosens T, Peerbooms JC, van Laar W, et al. Ongoing positive effect of platelet-rich plasma versus corticosteroid injection in lateral epicondylitis: a double-blind randomized controlled trial with 2-year follow-up. Am J Sports Med. 2011;39(6):1200-8. The authors report that with success being defined as a 25\% improvement in either VAS score or DASH score and no reintervention at 2-year follow-up, $65 \%$ of the PRP group and 35 $\%$ of the corticosteroid group had successful outcomes. This is an important clinical response that warrants recognition by practitioners who treat patients with lateral epicondylitis.

38. Creaney L, Wallace A, Curtis M, et al. Growth factor-based therapies provide additional benefit beyond physical therapy in resistant elbow tendinopathy: a prospective, single-blind, randomised trial of autologous blood injections versus platelet-rich plasma injections. Br J Sport Med. 2011;45(12):966-71.

39. Thanasas C, Papadimitriou G, Charalambidis C, et al. Plateletrich plasma versus autologous whole blood for the treatment of chronic lateral elbow epicondylitis: a randomized controlled clinical trial. Am J Sports Med. 2011;39(10):2130-4.

40. - Krogh TP, Fredberg U, Stengaard-Pedersen K, et al. Treatment of lateral epicondylitis with platelet-rich plasma, glucocorticoid, or saline: a randomized, double-blind, placebo-controlled trial. Am J Sports Med. 2013;41(3):625-35. The authors report that pain reduction was observed in all 3 groups, and there was no statistically significant difference in the primary outcome measure- Patient-Rated Tennis Elbow Evaluation (PRTEE) between the groups receiving either a PRP injection, saline injection, or corticosteroid injection at 3-month follow-up.36 It is important for providers who treat lateral epicondylitis to know of studies that refute PRP efficacy in addition to studies that support PRP efficacy.

41. Coombes BK, Bisset L, Vicenzino B. Efficacy and safety of corticosteroid injections and other injections for management of tendinopathy: a systematic review of randomised controlled trials. Lancet. 2010;376(9754):1751-67.

42. De Vos RJ, Weir A, Van Schie HTM, et al. Platelet-rich plasma injection for chronic Achilles tendinopathy. JAMA. 2010;303(2): 144-9.

43. De Vos RJ, Weir A, Tol JL, et al. No effects of PRP on ultrasonographic tendon structure and neovascularisation in chronic midportion Achilles tendinopathy. Br J Sport Med. 2011;45(5): 387-92.

44. - De Jonge S, de Vos RJ, Weir A, et al. One-year follow-up of platelet-rich plasma treatment in chronic Achilles tendinopathy: a double-blind randomized placebo-controlled trial. Am J Sport Med. 2011;39(8):1623-29. The authors report that there was not statistically significant difference in VISA (Victorian Institute of Sport Assessment) score or ultrasonographic appearance of the PRP group compared to the saline injection group at 1 year follow-up. It is important for providers who treat Achilles tendinopathy to know studies that refute PRP efficacy in addition to studies that support PRP efficacy.

45. Kon E, Filardo G, Delcogliano M, et al. Platelet-rich plasma new clinical application: a pilot study for treatment of jumper's knee. Injury. 2009;40(6):598-603.

46. Filardo G, Kon E, Della Villa S, et al. Use of platelet-rich plasma for the treatment of refractory jumper's knee. Int Orthop. 2010;34(6):909-15.

47. De Almeida AM, Demange MK, Sobrado MF, et al. Patellar tendon healing with platelet-rich plasma: a prospective, randomized, controlled trial. Am J Sports Med. 2012;40(6):1282-8.

48. Lyras DN, Kazakos K, Verettas D, et al. The effect of plateletrich plasma gel in the early phase of patellar tendon healing. Arch Orthop Trauma Surg. 2009;129(11):1577-82.

49. - Vetrano M, Castorina A, Vulpiani MC, et al. Platelet-rich plasma versus focused shock waves in the treatment of jumper's knee in athletes. Am J Sports Med. 2013;41(4):795-803. The authors report that there was statistically significant improvement in the $P R P$ group relative to the ECSW group at 6 month and 12 month follow-up using the VISA-P as an outcome measure. This is important to know for providers that treat patellar tendinopathy.

50. Mautner K, Colberg RE, Malanga G, et al. Outcomes after ultrasound-guided platelet-rich plasma injections for chronic tendinopathy: a multicenter retrospective review. PM R. 2013;5(3):169-75.

51. Bergeson AG, Tashjian RZ, Greis PE, et al. Effects of plateletrich fibrin matrix on repair integrity of at-risk rotator cuff tears. Am J Sports Med. 2012;40(2):286-93.

52. Rodeo Sa, Delos D, Williams RJ, et al. The effect of platelet-rich fibrin matrix on rotator cuff tendon healing: a prospective, randomized clinical study. Am J Sports Med. 2012;40(6):1234-41.

53. Jo $\mathrm{CH}$, Kim JE, Yoon $\mathrm{KS}$, et al. Does platelet-rich plasma accelerate recovery after rotator cuff repair? A prospective cohort study. Am J Sports Med. 2011;39(10):2082-90.

54. - Kesikburun S, Tan AK, Yilmaz B, et al. Platelet-rich plasma Injections in the treatment of chronic rotator cuff tendinopathy: a randomized controlled trial with 1-year follow-up. Am J Sports Med. 2013;41(11):2609-16. The authors report that in patients who received a single PRP injection or a single saline injection for partial thickness rotator cuff tears or rotator cuff tendinopathy, there were no statistically significant differences in the WORC score between the two treatment groups at 3-week, 6week, 12-week, 24-week or 1-year follow-up. It is important for practitioners who treat these conditions to be aware of this study that demonstrates no efficacy of a single PRP injection treatment.

55. - Rha D, Park G-Y, Kim Y-K, et al. Comparison of the therapeutic effects of ultrasound-guided platelet-rich plasma injection and dry needling in rotator cuff disease: a randomized controlled trial. Clin Rehabil. 2013;27(2):113-22. The authors report that 
patients with rotator cuff tendinosis or partial thickness tears treated with 2 serial PRP injections had statistically significant improvement relative to patients treated with 2 serial dry needling procedures at 2-week follow-up, at 3-month follow-up and at 6-month follow-up using the Shoulder Pain and Disability Index. It is important for providers who treat rotator cuff tendinopathy or partial thickness rotator cuff tears to be aware that 2 serial injections have demonstrated efficacy.

56. Finnoff JT, Fowler SP, Lai JK, et al. Treatment of chronic tendinopathy with ultrasound-guided needle tenotomy and plateletrich plasma injection. PM R. 2011;3(10):900-11.

57. Hróbjartsson A, Gøtzsche PC. Placebo interventions for all clinical conditions. Cochrane database Syst Rev. 2010;1:CD003974.

58. Murray MM, Spindler KP, Devin C, et al. Use of a collagenplatelet rich plasma scaffold to stimulate healing of a central defect in the canine ACL. J Orthop Res. 2006;24(4):820-30.

59. Silva A, Sampaio R. Anatomic ACL reconstruction: does the platelet-rich plasma accelerate tendon healing? Knee Surg Sports Traumatol Arthrosc. 2009;17(6):676-82.

60. Sánchez M, Anitua E, Azofra J, et al. Ligamentization of tendon grafts treated with an endogenous preparation rich in growth factors: gross morphology and histology. Arthroscopy. 2010;26(4):470-80.

61. Podesta L, Crow SA, Volkmer D, et al. Treatment of partial ulnar collateral ligament tears in the elbow with platelet-rich plasma. Am J Sports Med. 2013;41(7):1689-94.

62. Sampson S, Reed M, Silvers H, et al. Injection of platelet-rich plasma in patients with primary and secondary knee osteoarthritis: a pilot study. Am J Phys Med Rehabil. 2010;89(12):961-9.

63. Kon E, Buda R, Filardo G, et al. Platelet-rich plasma: intraarticular knee injections produced favorable results on degenerative cartilage lesions. Knee Surg Sports Traumatol Arthrosc. 2010;18(4):472-9.

64. Halpern B, Chaudhury S, Rodeo Sa, et al. Clinical and MRI outcomes after platelet-rich plasma treatment for knee osteoarthritis. Clin J Sport Med. 2013;23(3):238-9.

65. Jang S-J, Kim J-D, Cha S-S. Platelet-rich plasma (PRP) injections as an effective treatment for early osteoarthritis. Eur J Orthop Surg Traumatol. 2013;23(5):573-80.
66. Gobbi A, Karnatzikos G, Mahajan V, et al. Platelet-rich plasma treatment in symptomatic patients with knee osteoarthritis: preliminary results in a group of active patients. Sports Health. 2012;4(2):162-72.

67. Kon E, Mandelbaum B, Buda R, et al. Platelet-rich plasma intraarticular injection versus hyaluronic acid viscosupplementation as treatments for cartilage pathology: from early degeneration to osteoarthritis. Arthroscopy. 2011;27(11):1490-501.

68. Spaková T, Rosocha J, Lacko $M$, et al. Treatment of knee joint osteoarthritis with autologous platelet-rich plasma in comparison with hyaluronic acid. Am J Phys Med Rehabil. 2012;91(5):411-7.

69. Sánchez M, Anitua E, Azofra J, et al. Intra-articular injection of an autologous preparation rich in growth factors for the treatment of knee OA: a retrospective cohort study. Clin Exp Rheumatol. 2008;26(5):910-3.

70. - Cerza F, Carnì S, Carcangiu A, et al. Comparison between hyaluronic acid and platelet-rich plasma, intra-articular infiltration in the treatment of gonarthrosis. Am J Sports Med. 2012;40(12):2822-7. The authors report that patients who received 4 weekly PRP injections had statistically significant increased improvement in WOMAC score at week 24 relative to patients who received 4 weekly hyaluronic acid (HA) injections.61 There was also a statistically significant difference between grade III gonarthrosis treated with ACP and that treated with HA at week 12 and at week 24, with a noticeable improvement that was greater in the patients treated with ACP $(P<0.001)$. This improvement with 4 serial $P R P$ injections relative to $4 \mathrm{HA}$ injections is important to know for practitioners to treat patients with knee arthritis.

71. Sánchez M, Guadilla J, Fiz N, et al. Ultrasound-guided plateletrich plasma injections for the treatment of osteoarthritis of the hip. Rheumatology (Oxford). 2012;51(1):144-50.

72. Mei-Dan O, Carmont MR, Laver L, et al. Platelet-rich plasma or hyaluronate in the management of osteochondral lesions of the talus. Am J Sports Med. 2012;40(3):534-41.

73. Sanchez M, Anitua E, Andia I. Application of autologous growth factors on skeletal muscle healing. In: 2nd world congress on regenerative medicine. Poster presentation; 2005. p. 1. 